\title{
Recital dos Estudantes de Medicina da Universidade de São Paulo (REMUSP): descrição de um projeto musical de gestão, comunicação e relacionamento dos acadêmicos de medicina da Faculdade de Medicina da Universidade de São Paulo
}

\author{
"Recital dos Estudantes de Medicina da Universidade de São \\ Paulo (REMUSP)": description of a project of music, management, \\ communication and relationship of medical students of \\ "Faculdade de Medicina da Universidade de São Paulo"
}

\author{
Leandro Ryuchi luamoto', Rachel Emy Straus Takahashi², Augusto \\ Quaresma Coelho ${ }^{3}$, Ernesto Sasaki Imakuma ${ }^{4}$
}

\begin{abstract}
luamoto LR, Takahashi RES, Coelho AQ, Imakuma ES. Recital dos Estudantes de Medicina da Universidade de São Paulo (REMUSP): descrição de um projeto musical de gestão, comunicação e relacionamento dos acadêmicos de medicina da Faculdade de Medicina da Universidade de São Paulo / Recital dos Estudantes de Medicina da Universidade de São Paulo (REMUSP): description of a project of music, management, communication and relationship of medical students of "Faculdade de Medicina da Universidade de São Paulo". Rev Med (São Paulo). 2012 jul.-set.;91(3):198-201.
\end{abstract}

RESUMO: Recentemente tem se discutido muito sobre a formação médica dos acadêmicos das faculdades de medicina. A humanização é um aspecto que influencia a formação de um profissional médico com aptidão para lidar com seres humanos. Estudantes da Faculdade de Medicina da Universidade de São Paulo (FMUSP) com interesse em música se reuniram e formaram um grupo que realiza apresentações musicais em um recital. Assim foi criado o Recital dos Estudantes de Medicina da Universidade de São Paulo (REMUSP). Os membros do REMUSP realizam ensaios no Teatro da Faculdade de Medicina da USP de quatro a cinco horas por semana, aproximadamente, organizados através de grupos de Facebook e e-mail. No ano de 2011, durante a sua primeira apresentação, os membros do REMUSP executaram peças predominantemente do estilo clássico e popular, como: Canon In D - Johann Pachelbel, Ave Maria - Johann Sebastian Bach, Solfeggietto - C.P.E. Bach, Can You Feel The Love Tonight? - Elton John, New York, New York - Frank Sinatra, Kiss From a Rose - Seal etc. Atualmente, o REMUSP procura estimular a cooperatividade e organização nas apresentações musicais, sendo que os ensaios são cada vez mais preenchidos por peças tocadas em conjuntos, em detrimento das individuais, visando à integração e ao aperfeiçoamento da comunicação e organização de um grupo musical composto por futuros médicos mais humanizados.

DESCRITORES: Humanização da assistência; Gestão em saúde; Música; Medicina; Musicoterapia.

\begin{abstract}
Recently there has been an important discussion about the training of medical students from medical colleges. Humanization is an aspect that influences the formation of a medical professional with the ability to deal with human beings. Students of the Faculty of Medicine, University of São Paulo (USP) interested in music came together and formed a group that performs in a musical recital. Thus was created the Recital Students of Medicine, University of São Paulo (REMUSP). Members of REMUSP perform musical rehearsals at the Theatre, Faculty of Medicine, USP, four to five hours per week, approximately, organized through Facebook groups and e-mail. In 2011, during their first presentation, members of the REMUSP performed predominantly classic style and popular as: Canon In D - Johann Pachelbel, Ave Maria - Johann Sebastian Bach, Solfeggietto - CPE Bach, Can You Feel The Love Tonight? - Elton John, New York, New York - Frank Sinatra, Kiss From a Rose - Seal etc. Currently, the demand REMUSP stimulate cooperatively and organization in musical performances, and musical rehearsals are increasingly filled by pieces to be played by a group, at the expense of the individual, aiming at integration and improvement of communication and organization of a musical group composed of future doctors more humanized.
\end{abstract}

KEYWORDS: Humanization of assistance; Health management; Music; Medicine; Music therapy.

Recital dos Estudantes de Medicina da Universidade de São Paulo (REMUSP) - Comissão de Cultura e Extensão Universitária - Faculdade de Medicina da Universidade de São Paulo.

1. Graduando em Medicina pela Faculdade de Medicina da Universidade de São Paulo e Diretor Geral do III REMUSP.

2. Graduanda em Medicina pela Faculdade de Medicina da Universidade de São Paulo e Diretora Geral do II REMUSP),

3. Graduando em Medicina pela Faculdade de Medicina da Universidade de São Paulo e Diretor do II REMUSP.

4. Médico Residente em Cirurgia pela Faculdade de Medicina da Universidade de São Paulo e Membro do REMUSP.

Endereço para correspondência: Faculdade de Medicina da Universidade de São Paulo. Av. Dr. Arnaldo, 455 - Subsolo - Pacaembu, São Paulo, SP - CEP 01246-000. 


\section{INTRODUÇÃO}

A formação médica tem como característica importante a multidisciplinaridade. O estudante de medicina atravessa seis anos assistindo a aulas teóricas sobre todas as áreas médicas e aprendendo a prática clínica. Além disso, necessita de trabalhar em estágios diversos no hospital e atende a um enorme número de pacientes. Durante sua formação, médicos também aprendem a compreender o indivíduo de forma mais holística. O paciente não é apenas um fígado doente ou um nervo cortado, é um ser com família, que aprecia arte, que teme a dor e a morte. Esta visão de caráter humanístico começou a se tornar mais presente nos cursos de graduação médica nas últimas décadas ${ }^{1}$.

A humanização é aspecto que influencia a formação de um profissional médico com aptidão para lidar com seres humanos ${ }^{2}$. A Faculdade de Medicina da Universidade de São Paulo (FMUSP) oferece diversas disciplinas de humanidades no currículo nuclear e complementar, como Bases Humanísticas da Medicina. Assim, acredita-se que estes médicos estão mais reparados para se comunicar, ser mais compreensivos com o paciente e mais preparados para tomar decisões éticas ${ }^{3}$.

A formação holística envolve o estudo de humanidades médicas que tem por objetivo aumentar a sensibilidade e facilitar a empatia pelo paciente ${ }^{4}$. Arte, literatura, filosofia, dança, escultura, fotografia, cinema e música são consideradas humanidades médicas ${ }^{5}$. A música é arte presente em inúmeras culturas e é potente forma de expressão de emoções. A medicina possui relação histórica com a música. Os compositores clássicos Hector Berlioz e Alexander Borodin estudaram medicina ${ }^{1,6}$. Atualmente, podese observar música relacionada à medicina através de orquestras de médicos, a exemplo da Orquestra Filarmônica dos Médicos do Hospital Israelita Albert Einstein, da musicoterapia como disciplina médica e de pesquisas de controle de estresse através de música ${ }^{7,8,9}$.

Na FMUSP, um grupo de alunos busca fortalecer o seu vínculo com a música. Este grupo, o Recital dos Estudantes de Medicina da Universidade de São Paulo (REMUSP), completará um ano de existência neste ano. O presente trabalho se propõe a descrever as atividades do REMUSP e discutir as vantagens da existência de um espaço musical no curso de medicina.

\section{OBJETIVOS}

Descrever as atividades de um grupo musical composto por acadêmicos de medicina da FMUSP e discutir as vantagens da existência de um espaço musical no curso de medicina.

\section{Estratégias de atuação}

Os encontros musicais ocorrem uma vez a cada semana no Teatro da FMUSP com duração aproximadamente de 4 a 5 horas. Os membros começam a ensaiar as músicas tanto individualmente quanto coletivamente, depois de serem definidos os subgrupos e as peças musicais a serem realizadas. O grupo de facebook e de e-mail foram criados para facilitar a organização dos encontros do REMUSP. Nestes, a equipe que se compromete a colaborar se subdivide por afinidade de gosto musical e procura alguma peça musical que agrade às preferências musicais do subgrupo.

Cada peça selecionada é estudada de forma que seja possível sua execução obedecendo à métrica, sincronia, melodia e dinâmica. $O$ arranjo musical é elaborado a partir da melodia original. A maioria dos arranjos é criado pelos próprios membros, baseandose em interpretações realizadas por autores que lançam músicas na mídia, por exemplo no Youtube, CDs e DVDs de shows e concertos. Desta forma, novos instrumentos são incorporados ao arranjo.

Os instrumentos utilizados nos ensaios são elétricos e acústicos. À exceção do piano, os instrumentos acústicos são propriedades dos próprios membros, e consistem em violino, violão, gaita e bateria. Os aparelhos elétricos consistem em guitarra, violão elétrico, baixo, amplificadores, teclado e microfone. Este último é propriedade da FMUSP.

\section{RESULTADOS}

Na primeira apresentação realizada em novembro de 2011, os membros do REMUSP executaram peças do gênero clássico, popular e rock: Canon em D - J. Pachabel; Friozinha da Manhã - Z. Gramini; Ave Maria - J. S. Bach; Solfegietto No. 2 - C. P. E. Bach; Aria em G - J. S. Bach; Noturno em C\# Menor Op. 27 No. 1 - F. Chopin; Can you feel the love tonight - E. John; New York, New York - J. Kander e F. Ebb; More than words - G.Cherone e N. Bettencourt; Apoteose I. Izzo; Imagine - J. Lennon; Lótus Láctea-Y. Regis, Start - Depapepe; Habanera de Carmen - Z. Bizzet; Cenas do Oeste No. 1 - R. Schuman; Dança da fada de açúcar, Suíte do Quebra Nozes - P. I. Tchaicovky; 3ํㅡㄴ Movimento da Sonata em C Menor Op. 13 - L. Van Beethoven; Partita No.2 em D Menor - J. S. Bach; Valsa Brilhante No. 1 - F. Chopin; Preludio No. 5 - S. Rachmaninov; Me and Bobby McGee - K. 
Kristofferson; Valerie - A. Winehouse; Kiss from a Rose - Seal.

No ano de 2012, a equipe aumentou de 16 para 49 membros.

Observando os encontros e ensaios musicais no ano de 2012, observou-se uma tendência cada vez maior de formar subgrupos de quatro ou cinco pessoas, em detrimento das apresentações individuais. Este ano, sete subgrupos contam com mais de quatro pessoas.

\section{DISCUSSÃO}

A comunicação entre os membros se mostra um dos aspectos mais importantes. Nos departamentos da faculdade, uma das ferramentas de comunicação mais eficientes é a secretária. Faz parte de sua função organizar horários, informar sobre as atividades, marcar reuniões, organizar a burocracia. O REMUSP lançou mão de uma rede social para se comunicar. Assim como nos departamentos, organizar suas atividades é impensável sem o intercâmbio de ideias. Atualmente, o facebook e o grupo de emails são acessados pela internet, que hoje é veículo mais utilizado para fluxo de informações. O facebook se mostrou uma plataforma eficiente para divulgação de ideias para arranjos musicais, compartilhamento de partituras musicais, marcação de ensaios. Dessa forma, o grande número de pessoas que passou a integrar o REMUSP pode ser consequência da facilidade de divulgação de informações e eficiência da comunicação atingida pelo grupo.

A comunicação também foi chave para o êxito do trabalho em equipe. Assim como para se concluir com sucesso a terapia de um paciente complexo é necessário ser flexível, ser receptivo a ideias diferentes e enxergar o ponto de vista de profissionais de diferentes áreas de atuação, como fonoaudiologia, fisioterapia, enfermagem, as músicas apresentadas pelo REMUSP também requereram o uso de diversas destas competências interpessoais. Na medida em que os subgrupos ensaiaram em conjunto, os membros passaram a trabalhar sua comunicação e expandiram sua vivência e conhecimento na área de humanidades. Desta forma, acredita-se que o estudante lide melhor com a diversidade cultural e seja capaz de construir uma boa relação médicopaciente. As mesmas competências garantiram uma apresentação em níveis satisfatórios ao público. Esta satisfação se verificou nos depoimentos de muitos espectadores que declararam ter $\mathrm{o}$ interesse em aprender a tocar um instrumento ou em aperfeiçoar a técnica de um que já toca.

Foi verificado que a prática musical estimula um aperfeiçoamento da cognição, raciocínio, concentração e coordenação motora. Além disso, o processo de reinterpretação das peças musicais pelo grupo contribui para o fortalecimento da criatividade dos membros. Ao juntar tais benefícios com o trabalho em grupo dentro de uma instituição (REMUSP), o futuro médico encontra nesse grupo uma maneira de desenvolver habilidades de organização, comunicação, raciocínio lógico e relaxamento, importantes não só na carreira médica, mas em todas as que implicam em certo "impacto estressante" sobre os profissionais da área.

A prática musical é também uma maneira de relaxamento. A música já está sendo utilizada como ferramenta terapêutica por médicos para tratamento de estresse. A musicoterapia, embora não utilizada de maneira rotineira nos hospitais, possui evidências de sua ação na literatura ${ }^{10}$. O curso de graduação de medicina é longo e em diversos momentos desgastante, principalmente emocionalmente, pelo contato do aluno com o sofrimento e a morte. A música ameniza sentimentos negativos. Muitas pessoas escutam música ou interpretam uma para se sentir melhor. $O$ REMUSP tem um potencial desconhecido e não estudado em gerenciar estresse e prevenir transtornos de humor, como o transtorno depressivo.

$O$ fato de haver ingressado no REMUSP 33 novos membros indicam o quanto muitos estudantes necessitam de um tempo e espaço para se dedicarem a um lazer e aliarem-se juntamente a um círculo social, em que ao mesmo tempo ganhariam muito mais do esperam em se tratando de experiência nas relações humanas. A diversidade de gêneros musicais apresentados (clássico, popular e rock) possibilitou aos integrantes do grupo explorar suas habilidades em diversos estilos de música e desfazer a imagem do REMUSP como um grupo que se restringe a apresentar músicas de estilo clássico, como na maioria dos recitais.

\section{CONCLUSÕES}

O REMUSP é um grupo formado por estudantes de medicina que abre espaço musical eclético para interessados em música. Tal espaço também é utilizado para o aperfeiçoamento de competências de trabalho em equipe. Esta se comunica de maneira eficiente através de grupos no Facebook e e-mail. $O$ REMUSP tem um potencial ainda não estudado de gerenciar estresse, melhorar a comunicação com o paciente e incutir o interesse em aprender a tocar ou aperfeiçoar a habilidade em um instrumento musical. 


\section{REFERÊNCIAS}

1. Weisz GM, Albury WR. The medico-artistic phenomenon and its implications for medical education. Med Hypothes. 2010;74(1):169-73. doi:10.1016/j.mehy.2009.07.013.

2. Caprara A, Franco ALS. A relação paciente-médico: para uma humanização da prática médica. Cad Saúde Publica. 1999;15(3):647-54.

3. Dellasega C, Milone-Nuzzo P, Curci KM, Ballard JO, Kirch DG. The humanities interface of nursing and medicine. J Prof Nurs. 2007;23(3):174-9.

4. Gordon J. Medical humanities: to cure sometimes, to relieve often, to comfort always. Med J Aust. 2005; 182(1):5-8.

5. McLellan L, McLachlan E, Perkins L, Dornan T. Music and health. Phenomenological investigation of a medical humanity. Adv Health Sci Educ. 2012 March 7. DOI:10.1007/s10459-012-9359-y

6. Lasseron M. Music and medicine. Med Humanities. 2008;34(2):118-9. doi:10.1136/jmh. 2008.

7. Panacioni GFA, Zanini CRO. Musicoterapia na promoção da saúde e controle do estresse: um projeto em andamento. Disponível em: http://www.sbpcnet. org.br/livro/63ra/conpeex/mestrado/trabalhosmestrado/mestrado-graziela-franca.pdf

8. Silva FO, Ferreira EABF, Miranda AMC. Musicoterapia na prevenção e/ou diminuição do estresse psicofisiológico durante a hospitalização: um estudo com pacientes entre 10 e 21 anos [monografia]. Goiânia: Universidade Federal de Goiás, Curso de Graduação em Musicoterapia; 2005.

9. Silva FO, Craveiro de Sá LC. Experiências de Re-criação Musical e Composição Musical em Musicoterapia: estratégias de enfrentamento ao estresse? Disponível em: http://www.ufg.br/ conpeex/2006/porta_arquivos/outras/1798854FernandaOrtinsSilva.pdf.

10. Chiarelli LKM, Barreto SJ. A música como meio de desenvolver a inteligência e a integração do ser. Rev Recre@ @rte. 2005 jun.;(3). Disponível em: http://www. iacat.com/revista/recrearte/recrearte03.htm 